\title{
TESTE DE ACEITABILIDADE SENSORIAL DE QUEIJO DE COALHO COM BAIXO TEOR DE GORDURA E ENRIQUECIDO COM FERRO *
}

\author{
TIANE FRANCO BARROS MANGUEIRA ** \\ ANTONIO EUSTAQUIO RESENDE TRAVASSOS *** \\ RICARDO TARGINO MOREIRA ****
}

\begin{abstract}
Avaliou-se a aceitabilidade de queijo de coalho, com baixo teor de gordura e enriquecido com ferro. Foram elaborados dois tipos de queijo (desnatado e parcialmente desnatado) enriquecidos com dois tipos de ferro. Os queijos parcialmente desnatados QLPD (aproximadamente 2\% de gordura) foram adicionados de ferro na forma de sal (sulfato ferroso amoniacal - QLPD-Fs) e na forma de aminoácido quelato ("Ferrochel" - QLPD-Fq). Os queijos elaborados com leite desnatado [QLD] foram adicionados de ferro na forma de sal (sulfato ferroso amoniacal - QLD-Fs) e na forma de aminoácido quelato ("Ferrochel" - QLD-Fq). As amostras de queijo de coalho foram analisadas mediante escala hedônica, sendo avaliados atributos como aroma, aspecto geral, cor, sabor e textura. Verificou-se ainda, a existência ou não de sabor estranho e a intensão de compra do produto. Os dados obtidos foram submetidos à ANOVA e quando necessário ao teste de Tukey $(5 \%$ de probabilidade). As médias atribuídas pelos provadores indicaram preferência pelos queijos QLPD-Fs e QLPD-Fq, os quais provavelmente comprariam. Observou-se sabor estranho de forma mais acentuada nos queijos QLD-Fs e QLD-Fq. As amostras QLPD-Fs e QLPD-Fq apresentaram maior percentual de valores na faixa positiva de compra, e os queijos QLD-Fs e QLD-Fq menor aceitação e menor interesse de compra.
\end{abstract}

PALAVRAS-CHAVE: QUEIJO DE COALHO-ANÁLISE SENSORIAL; QUEIJO-FERRO; QUEIJO-GORDURA.

* Parte da Dissertação de Mestrado em Ciência e Tecnologia de Alimentos, do primeiro autor.

** Mestre em Ciência e Tecnologia, Departamento de Tecnologia Química e de Alimentos, Centro de Tecnologia, Universidade Federal da Paraíba (UFPB), Campus I, João Pessoa/PB. (e-mail: tianemangueira@bol.com.br).

*** Prof. Doutor, Departamento de Tecnologia Rural, Centro de Formação de Tecnólogos, UFPB, Campus IV, Bananeiras (PB) e Professor Associado ao Departamento de Tecnologia Química e de Alimentos do Centro de Tecnologia, UFPB/Campus I, João Pessoa/PB. (e-mail: eustcft@cft.ufpb.br e/ou cpgcta@ct.ufpb.br).

**** Prof. Mestre, Departamento de Tecnologia Rural, Centro de Formação de Tecnólogos, UFPB/Campus IV, Bananeiras/PB. (e-mail: ricardo@cft.ufpb.br). 


\section{INTRODUÇÃO}

O queijo de coalho constitui-se em derivado do leite de grande popularidade, aceitação, consumo e produção na região Nordeste do Brasil. Seu nome deve-se ao fato de ter sido tradicionalmente elaborado com leite coagulado pela ação do coalho animal. Pedaços de estômago de pequenos animais (mocó, preá, cabritos, ovelhas e bezerros), que devidamente preparados são conhecidos por abomasun ou coagulador (2). Apesar de sua importância econômica e grande popularidade na região, a fabricação de queijo de coalho não conta com tecnologia apropriada para a melhoria de sua qualidade. É necessário que o processo tradicional, geralmente artesanal e a partir de leite cru, acompanhe a evolução das técnicas queijeiras mediante modificações já utilizadas na tecnologia de outros queijos nacionais (8).

O queijo apresenta elevado valor nutritivo devido grande concentração de proteínas, sais minerais e vitaminas. Trata-se de produto muito rico em fósforo e cálcio (5, 6 e 14). Todavia, é pobre em ferro (4) e sua carência pode comprometer o crescimento, atrasar o desenvolvimento cerebral e diminuir a resistência às infecções (9).

De acordo com LOTUFO (9), na alimentação normal, as principais fontes de ferro são a carne e o feijão. Portanto, seria necessária a ingestão de grande quantidade desses dois alimentos para se alcançar a Ingestão Diária Recomendada (IRD) do mineral. De acordo com a Resolução CNNPA 12/78 (3), a dose diária de ferro recomendada para adultos em estado físico normal é de 10 a $15 \mathrm{mg}$.

O aumento no teor de ferro do leite, utilizado para fabricação de queijos, poderia tornar o produto mais completo e suprir a deficiência de ferro que atinge parte da população.

A cada dia buscam-se alimentos que não prejudiquem e a saúde, sendo crescente a preocupação dos consumidores com a qualidade nutricional de sua dieta. Entretanto, ainda é freqüente a ingestão excessiva de gordura saturada, como ocorre com a do leite.

Com o objetivo de aumentar a oferta de produtos que contribuam para dietas mais saudáveis foram desenvolvidos e testados, sensorialmente, queijos de leite de vaca com baixo teor de gordura (produto light) e enriquecidos em ferro. 


\section{MATERIAL E METÓDOS}

\subsection{MATÉRIA PRIMA}

Empregou-se leite de vaca, coletado em janeiro de 2001, proveniente do plantel de vacas mestiças zebu-holandês, pertencentes ao Campus IV da Universidade Federal da Paraíba (UFPB). O referido Campus está localizado no município de Bananeiras/PB, a aproximadamente $140 \mathrm{Km}$ da cidade de João Pessoa, na Microrregião do Brejo Paraibano.

\subsection{ELABORAÇÃO DOS QUEIJOS}

Os trabalhos foram conduzidos na Unidade de Pesquisa e Demonstração de Alimentos (UPEDA) e no Laboratório de Controle de Qualidade de Alimentos, pertencentes ao Centro de Formação de Tecnólogos de Bananeiras (CFT) da UFPB.

Após a recepção, o leite foi filtrado e pesado, sendo em seguida desnatado (desnatadeira mecânica) de forma parcial QLPD (com aproximadamente $2 \%$ de gordura, mediante Quadrado de Pearson) e total (QLD). Empregouse pasteurização lenta, a temperatura de 63 a $65^{\circ} \mathrm{C}$, durante 30 minutos. Em seguida o leite foi resfriado em água corrente até alcançar a temperatura de aproximadamente $35^{\circ} \mathrm{C}$.

Após a pasteurização foram adicionados ao leite a cultura láctea, o cloreto de cálcio a $50 \%$, e os dois tipos de ferro (sulfato ferroso amoniacal $\left[\mathrm{Fe}\left(\mathrm{NH}_{4}\right)_{2}\left(\mathrm{SO}_{4}\right)_{2} .6 \mathrm{H}_{2} \mathrm{O}\right] 150 \mathrm{mg} / 100 \mathrm{~mL}-$ Ferro em forma de sal e o mineral "Ferrochel" do Laboratório Albion (1) [Fe( $\left.\left.\mathrm{C}_{2} \mathrm{NO}_{2} \mathrm{H}_{2} \mathrm{R}\right)_{2} . \mathrm{H}_{2} \mathrm{O}\right](88 \mathrm{mg} / 100$ $\mathrm{mL}-$ Ferro em forma de aminoácido quelato. Por último juntou-se o coalho líquido enzimático de marca comercial "Há-la", na proporção de 7 a $8 \mathrm{~mL}$ para cada dez litros de leite.

O leite foi agitado para facilitar a distribuição do material adicionado e deixado em repouso durante 40 a 50 minutos para coagulação. Em seguida, efetuou-se o corte da coalhada, usando espátula de aço inoxidável.

Após a sedimentação total dos grãos de massa depositados no fundo do recipiente foi feita a dessoragem. Na coalhada obtida foi adicionado sal comum (na proporção de $0,2 \%$ de sal em relação ao peso da massa), procedendo-se a enformagem em recipientes retangulares de PVC com dessorador e realizada a prensagem em prensa mecânica. A seguir, os 
queijos foram levados à câmara de refrigeração a $10 \pm 2^{\circ} \mathrm{C}$, na qual permaneceram por 3 dias.

O queijo fabricado de formato retangular, apresentou $6,1 \mathrm{~cm}$ de altura, $8,4 \mathrm{~cm}$ de largura e $17 \mathrm{~cm}$ de comprimento, com peso variando entre 500 e 850 gramas.

\subsection{AMOSTRAS}

Foram elaborados dois tipos de queijo de leite de vaca, um com leite parcialmente desnatado e outro desnatado, ambos enriquecidos com dois tipos de ferro (totalizando quatro amostras). Os queijos elaborados com leite parcialmente desnatado (aproximadamente $2 \%$ de gordura) foram adicionados de ferro na forma de sal (Sulfato Ferroso Amoniacal - QLPDFs) e na forma de aminoácido quelato (Ferrochel-QLPD-Fq). Os queijos preparados com leite desnatado também foram adicionados de ferro na forma de sal (Sulfato Ferroso Amoniacal - QLD-Fs) e na forma de aminoácido quelato (Ferrochel-QLD-Fq).

\subsection{ANÁLISE SENSORIAL}

As avaliações sensoriais foram realizadas de acordo com MORAES (11), em cabines individuais, longe de ruídos e odores, em horários previamente estabelecidos (excluindo uma hora antes e duas horas após o almoço).

A aceitabilidade dos queijos foi determinada com participação de consumidores potenciais do produto. Utilizou-se escala hedônica estruturada mista de nove pontos ( 1 = desgostei extremamente; $5=$ nem gostei/nem desgostei; 9 = gostei extremamente), conforme STONE e SIDEL (13), para avaliar o produto quanto ao aroma, aspecto geral, intensidade da cor, intensidade do sabor e textura. Empregou-se escala hedônica de cinco pontos para a intenção de compra ( 1 = certamente não compraria; 3 = talvez comprasse/talvez não comprasse; 5 = certamente compraria) e a presença de sabor estranho ( 1 = nenhum sabor estranho; $3=$ sabor estranho moderadamente forte; 5 = sabor estranho forte).

Inicialmente foram entrevistados 110 provadores, com base na disponibilidade e interesse de participar dos testes sensoriais, sendo indagada a periodicidade de consumo do queijo de coalho. O painel de provadores foi constituído de 100 pessoas selecionadas, de ambos os sexos, de diferentes faixas etárias (variando de 15 a 55 anos) e não treinadas, incluindo professores, funcionários, alunos e estagiários do Centro de Formação de Tecnólogos. 
As amostras foram servidas em cubos uniformes de $1,0 \mathrm{~cm}$ por $1,0 \mathrm{~cm}$, em pratos descartáveis brancos, codificadas com números de três dígitos definidos de forma aleatória. O desenho experimental utilizado para controlar os efeitos de posição da amostra foi o de blocos completos balanceados conforme proposto por WAKELING e MACFIE (15).

As amostras foram servidas em temperatura ambiente, acompanhadas de biscoito água e sal e água mineral para remoção do sabor residual. Todas as amostras foram apresentadas simultaneamente e os provadores orientados para provar uma de cada vez da esquerda para direita. Juntouse o questionário para avaliação do quanto gostaram ou desgostaram do aroma, aspecto geral, cor, sabor e textura dos queijos.

Foi verificada a existência ou não de sabor estranho e qual seria esse sabor, sendo perguntado se os provadores comprariam ou não o produto.

\subsection{ANÁLISE ESTATÍSTICA DOS RESULTADOS}

Os resultados obtidos foram avaliados mediante Análise de Variância (ANOVA) e Teste de Tukey com o pacote estatístico SPSS (Statistical Package for the Social Sciences) (12) e gráfico Aranha de acordo com MONTEIRO (10).

\section{RESULTADOS E DISCUSSÃO}

Das 110 pessoas entrevistadas, 90,09\% (100) foram selecionadas para o teste sensorial do queijo de coalho. Quanto à periodicidade de consumo, $94 \%$ desses informaram consumir queijo de coalho de uma a quatro vezes por semana e $6 \%$ mais de cinco vezes por semana. Quanto à forma de consumo, $31 \%$ preferem frito na manteiga, $27 \%$ assado, $19 \%$ cru e $23 \%$ consomem o queijo de coalho das três formas.

$\mathrm{Na}$ Tabela 1 estão apresentadas as médias de aceitação aos atributos sensoriais para os dois tipos de queijo elaborados.

Não foi verificada diferença estatística significativa $(P<0,05)$ para o atributo sabor nos queijos avaliados, ao contrário das demais características (aroma, aspecto geral, cor e textura).

No atributo sabor, apesar dos queijos QLPD-Fs e QLPD-Fq diferirem em valores reais (obtendo média " 5 ") dos queijos QLD-Fs e QLD-Fq (média entre "5" e "6"), não houve diferença estatística $(P<0,05)$. 


\section{TABELA 1 - MÉDIAS DE ACEITAÇÃO DOS QUEIJOS DE COALHO DE LEITE PARCIALMENTE DESNATADO (QLPD) E DESNATADO (QLD) ENRIQUECIDOS COM FERRO EM FORMA DE SAL (Fs) OU AMINOÁCIDO QUELATO (Fq)}

\begin{tabular}{llllll}
\hline Amostra & Aroma & $\begin{array}{l}\text { Aspecto } \\
\text { geral }\end{array}$ & Cor & Sabor & Textura \\
\hline QLPD-Fs & $6,39^{\mathrm{bc} *}$ & $6,79^{\mathrm{b}}$ & $5,20^{\mathrm{b}}$ & $5,12^{\mathrm{a}}$ & $5,43^{\mathrm{b}}$ \\
QLD-Fs & $5,88^{\mathrm{a}}$ & $4,57^{\mathrm{a}}$ & $3,39^{\mathrm{a}}$ & $5,67^{\mathrm{a}}$ & $7,96^{\mathrm{c}}$ \\
QLPD-Fq & $6,55^{\mathrm{c}}$ & $7,02^{\mathrm{b}}$ & $5,13^{\mathrm{b}}$ & $5,14^{\mathrm{a}}$ & $4,84^{\mathrm{a}}$ \\
QLD-Fq & $5,95^{\mathrm{ab}}$ & $4,44^{\mathrm{a}}$ & $3,37^{\mathrm{a}}$ & $5,33^{\mathrm{a}}$ & $7,92^{\mathrm{c}}$ \\
\hline
\end{tabular}

* Médias na mesma coluna seguidas por letras diferentes diferem entre si pelo teste de Tukey $(P<0,05)$.

As modificações, tanto no teor de gordura, como no tipo de ferro adicionado, segundo os provadores, quase não alteraram o sabor do produto. ZHANG e MAHONEY (16) analisaram o sabor do queijo cheddar enriquecido com 10 a $20 \mathrm{mg}$ de ferro por $100 \mathrm{~g}$ de queijo na forma de $\mathrm{FeCl}_{3}$ e não encontraram diferença significativa $(\mathrm{P}<0,05)$ em relação a testemunha (queijo sem adição de ferro).

Com relação ao aroma, os queijos parcialmente desnatados (QLPD-Fs e QLPD-Fq) obtiveram médias entre "6" e "7" da escala hedônica que equivalem, respectivamente, aos conceitos "gostei ligeiramente" e "gostei moderadamente". Para o atributo aspecto geral, os queijos QLPD-Fs e QLPD-Fq receberam as maiores notas cuja média (próxima a "7") corresponde a "gostei moderadamente". As médias para o atributo aroma dos queijos QLD-Fs e QLD-Fq ficaram próximas a "6" que equivale a "gostei ligeiramente".

Com relação ao aspecto geral, os queijos QLPD-Fs e QLPD-Fq obtiveram as maiores médias (6,79 e 7,02 respectivamente) não apresentando diferença estatística ao nível de $5 \%$ de probabilidade. Os queijos QLD-Fs e QLD-Fq obtiveram as médias mais baixas (4,57 e 4,44 respectivamente) equivalentes ao conceito "desgostei ligeiramente". Possivelmente os provadores perceberam alteração no aspecto geral do produto da retirada parcial ou total da gordura.

Para o atributo cor os queijos QLPD-Fs e QLPD-Fq alcançaram média "5” 
e os queijos QLD-Fs e QLD-Fq obtiveram conceito "moderadamente mais clara que a ideal" (média 3). A retirada de quantidade maior de gordura, além de interferir na cor, modificou também a textura do produto. Os queijos QLD-Fs e QLD-Fq apresentaram-se mais firmes que os demais, obtendo média "8" (próximo do conceito máximo "extremamente firme").

A Figura 1 mostra a aceitação das amostras em relação aos atributos aroma, aspecto geral, cor, sabor e textura. Quanto maior a sobreposição das amostras, maior é a semelhança entre si e o eixo y representa a aceitação de cada atributo. Pode-se verificar que os dois tipos de queijo variaram com relação ao aspecto geral. Os queijos QLPD-Fs e QLPD-Fq apresentaram maiores valores médios na escala hedônica para esse parâmetro. O atributo cor alcançou maior valor médio (nota) para os queijos QLPD-Fs e QLPD-Fq.

\section{FIGURA 1 - VALORES MÉDIOS DOS ATRIBUTOS SENSORIAIS OBTIDOS PELO QLPD-Fs, QLPD-Fq, QLD-Fs E QLD-Fq}

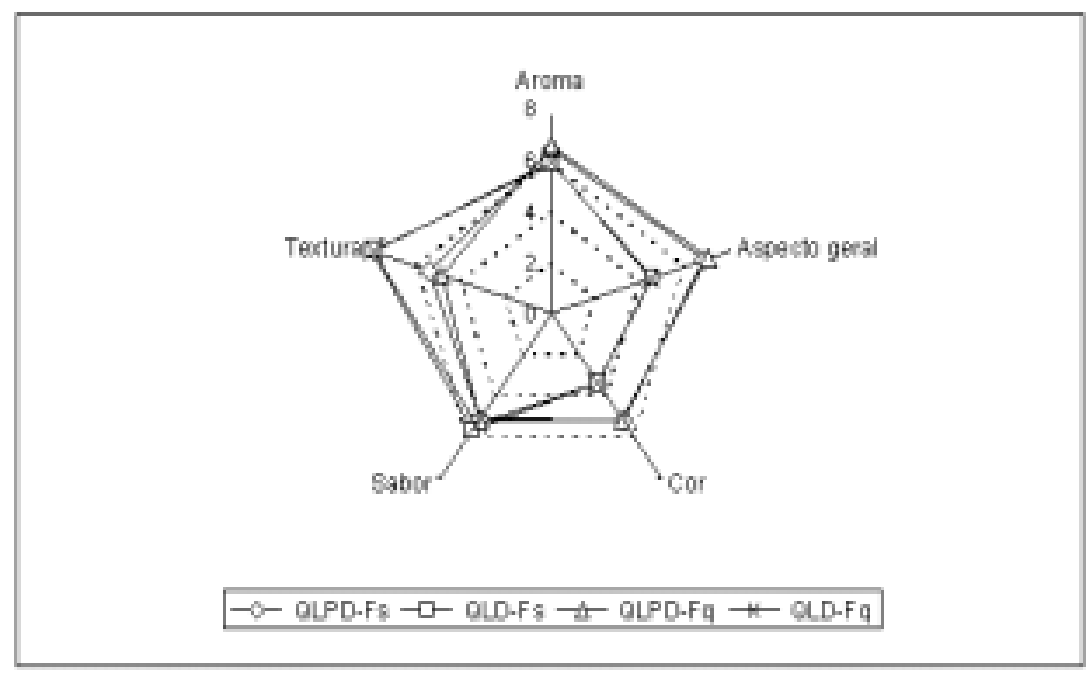

Com relação à presença de sabor estranho e intenção de compra houve diferença significativa $(P<0,05)$ entre os queijos estudados (Tabela 2). Entretanto, os queijos QLPD-Fs e QLPD-Fq não diferem entre si, assim como os queijos QLD-Fs e QLD-Fq mostraram-se iguais ao nível de 5\% de probabilidade. 
TABELA 2 - MÉDIAS DE ACEITAÇÃO DOS QUEIJOS DE COALHO DE LEITE PARCIALMENTE DESNATADO (QLPD) E DESNATADO (QLD) ENRIQUECIDOS COM FERRO EM FORMA DE SAL (Fs) OU AMINOÁCIDO QUELATO (Fq), EM RELAÇÃO À PRESENÇA DE SABOR ESTRANHO E A INTENÇÃO DE COMPRA

\begin{tabular}{lll}
\hline Amostra & $\begin{array}{l}\text { Presen a de sabor } \\
\text { estranho }\end{array}$ & Inten a o de compra \\
\hline QLPD-Fs & $1,57^{\mathrm{a} *}$ & $3,87^{\mathrm{b}}$ \\
QLD-Fs & $2,83^{\mathrm{b}}$ & $1,82^{\mathrm{a}}$ \\
QLPD-Fq & $1,59^{\mathrm{a}}$ & $4,07^{\mathrm{b}}$ \\
QLD-Fq & $2,78^{\mathrm{b}}$ & $1,80^{\mathrm{a}}$ \\
\hline
\end{tabular}

* Médias na mesma coluna seguidas por de letras diferentes diferem entre si pelo teste de Tukey $(P<0,05)$.

Na Figura 2 observa-se as pontuações atribuídas aos queijos, com relação à presença e intensidade de sabor estranho.

\section{FIGURA 2 - NÚMERO DE PROVADORES, EM PERCENTAGEM E AS RESPECTIVAS PONTUAÇÕES AFERIDAS À PRESENÇA DE SABOR ESTRANHO NOS QUEIJOS}

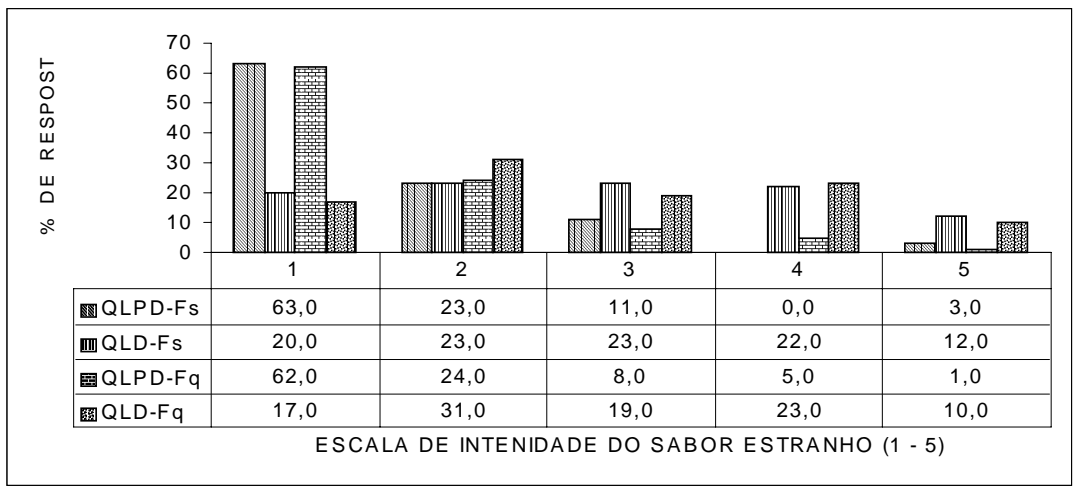

Os queijos QLPD-Fs e QLPD-Fq obtiveram notas referentes a "nenhum sabor estranho" (63\% e $62 \%$, respectivamente) e os queijos desnatados (QLD-Fs e QLD-Fq) notas 4 (forte) e 5 (muito forte). 
O sabor "azedo" foi identificado com maior freqüência e de forma mais acentuada no queijo QLPD-Fq (Tabela 3).

\section{TABELA 3- DISTRIBUIÇÃO DOS SABORES ESTRANHOS IDENTIFICADOS NOS QUEIJOS DE LEITE PARCIALMENTE DESNATADO (QLPD) E DESNATADO (QLD) ENRIQUECIDOS COM FERRO}

\begin{tabular}{|c|c|c|c|c|c|c|c|c|}
\hline \multirow{3}{*}{$\begin{array}{l}\text { Sabor } \\
\text { estranho } \\
\text { encontrado }\end{array}$} & \multicolumn{8}{|c|}{ Queijo } \\
\hline & \multicolumn{2}{|c|}{ QLPD-Fs } & \multicolumn{2}{|c|}{ Q LD-Fs } & \multicolumn{2}{|c|}{ Q LPD-Fq } & \multicolumn{2}{|c|}{ QLD-Fq } \\
\hline & $\mathbf{n}$ & $\%$ & $\mathbf{n}$ & $\%$ & $\mathbf{n}$ & $\%$ & $n$ & $\%$ \\
\hline $1 \mathrm{Amargo}$ & 4 & 10,5 & 11 & 15,5 & 4 & 11,4 & 10 & 14,9 \\
\hline $2 \mathrm{Met} /$ \#ico & 7 & 18,4 & 17 & 23,9 & 4 & 11,4 & 19 & 28,4 \\
\hline 3 Medicinal & 7 & 18,4 & 5 & 7,0 & 1 & 2,9 & 3 & 4,5 \\
\hline 4 Mofo & - & - & 3 & 4,2 & - & - & 5 & 7,5 \\
\hline 5 Azedo & 9 & 23,7 & 17 & 23,9 & 17 & 48,6 & 21 & 31,3 \\
\hline 6 Doce & - & - & - & - & - & - & - & - \\
\hline 7 Ran oso & 6 & 15,8 & - & - & 4 & 11,4 & - & - \\
\hline 8 A mido & 3 & 7,9 & 11 & 15,5 & 2 & 5,7 & 6 & 9,0 \\
\hline Outro & 2 & 5,3 & 7 & 9,9 & 3 & 8,6 & 3 & 4,5 \\
\hline TOTAL & 38 & 100,0 & 71 & 100,0 & 35 & 100,0 & 67 & 100,0 \\
\hline
\end{tabular}

$\mathrm{n}=$ Número de provadores que identificaram sabor estranho.

LIMA et al. (7) estudaram leite e iogurte enriquecido com ferro em forma de sal $\left(\mathrm{FeSO} 4.7 \mathrm{H}_{2} \mathrm{O}\right)$. Ambos mostraram-se aceitáveis nas dosagens de 1,0 e $0,7 \mathrm{mg} / 100 \mathrm{~g}$, respectivamente. Já o iogurte, com 1,0 mg/100 g não foi considerado aceitável em todas as avaliações (sabor adstringente, odor ligeiramente defeituoso e modificações na consistência).

Quanto à intenção de compra do produto pelos consumidores (Figura 3) as amostras dos queijos QLPD-Fs e QLPD-Fq apresentaram maior percentual de valores na faixa positiva de compra $(41 \%$ e $31 \%$ respectivamente). Verificou-se pouco interesse de compra para os queijos QLD-Fs (nota 1) e QLD-Fq (nota 2).

As médias atribuídas pelos provadores indicaram preferência na maioria dos atributos, pelos queijos parcialmente desnatados QLPD-Fs e QLPD$\mathrm{Fq}$, os quais provavelmente comprariam. 


\section{FIGURA 3 - MÉDIA DAS NOTAS ATRIBUÍDAS PELOS PROVADORES, COM RELAÇÃO À INTENÇÃO DE COMPRA DOS QUEIJOS ESTUDADOS}

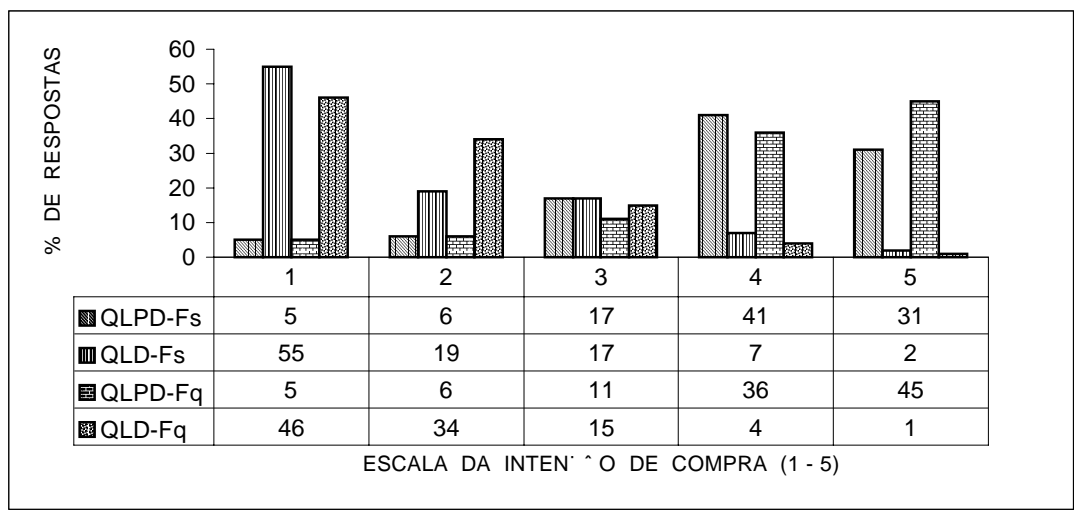

\section{CONCLUSÃO}

Os queijos de coalho elaborados com leite parcialmente desnatado (QLPDFs e QLPD-Fq) obtiveram maior aceitabilidade e despertaram maior interesse de compra.

É possível utilizar o ferro em forma de sal (sulfato ferroso amoniacal) ou aminoácido quelato (Ferrochel) no enriquecimento de queijo de coalho.

\section{Abstract}

SENSORY ACCEPTABILITY TEST OF BRAZILIAN COMMERCIAL "COALHO" CHEESE WITH LOW FAT CONTENT AND ENRICHED WITH IRON

The acceptability of "coalho" cheese with low fat content and enriched with iron were evaluated. Two types of cheese enriched with two types of iron were elaborated. The partially skimmed cheese (approximatelly $2 \%$ of fat) were enriched with iron in salt form (amoniacal iron sulfate - QLPD-Fs) and in the form of chelate amino acid ("Ferrochel" - QLPD-Fs). The cheese elaborated with skimmed milk were enriched with iron in salt form (amoniacal iron sulfate - QLD-Fs) and in the form of chelate amino acid ("Ferrochel" - QLD-Fq). The samples of "coagulum" cheese were analyzed through the Hedonic Scale method, where the attributes as aroma, general aspect, color, flavor and texture of the cheeses were appraised. The existence or not of strange flavor, and which flavor it would be as well as if the tasters would buy the product or not were still analyzed. The obtained data were submitted to the ANOVA and, when necessary, 
Tukey test at $5 \%$ of probability. The averages attributed by the tasters indicated preference for the cheeses QLPD-Fs and QLPD-Fq, which they would probably buy. With regard to the strange flavor, it was shown with great frequency the cheeses QLD-Fs and QLD-Fq. The samples QLPD-Fs and QLPD-Fq presented greater percentage of values in the positive strip of purchase, and the cheeses QLD-Fs and QLD-Fq an inferior acceptance and smaller purchase interest.

KEYWORDS: "COALHO" CHEESE-SENSORIAL ANALYSIS; IRON-CHEESE; FATCHEESE.

\section{REFERÊNCIAS}

1 ALBION LABORATORIES. Fortificação de alimentos. São Paulo, 2000.

2 AQUINO, F.T.M. Produção do queijo de coalho no Estado da Paraíba: acompanhamento das características fisico-química do processamento. João Pessoa, 1983. 81 p. Dissertação (Mestrado em Ciência e Tecnologia de Alimentos) - Universidade Federal da Paraíba.

3 ABIA. Associação Brasileira das Indústrias da Alimentação. Compêndio da legislação de alimentação: alimentos enriquecidos (Resolução da Comissão Nacional de Normas e Padrões para Alimentos - CNNPAMS n.12/78): sub-item 12/46. São Paulo, 1978. (Reunião 3, 7.32 (78)).

4 ATHERTON, H.V.; NEWLANDER, J.A. Chemistry and testing of dairy products. $4^{\text {th }}$ ed. [New York]: Avi, 1982. $396 \mathrm{p}$.

5 BASTOS, O. de S.; LIMA, M. de F.V.; LIMA, B.G.; ARAÚJO, Z.M.G.C. Avaliação físico-química do leite esterilizado desnatado comercializado em São Luís - MA. In: CONGRESSO BRASILEIRO DE CIÊNCIA E TECNOLOGIA DE ALIMENTOS, 17., 2000. Fortaleza. Anais... Fortaleza: SBCTA, 2000. (Ref. 3.47).

6 ESCOLANOVA LOURENÇO CASTANHO. Queijos. Disponível em:<http:/ /www.lourençocastanho.com.br/proj_trab/profmame2.htm>. Acesso em: 9 maio 2001.

7 LIMA, L.B.; PÉREZ, S.B.; BOLAÑOS, M.J. de O. et al. Leche fluida y yogur natural enriquecidos con hierro. Alimentaria, v. 63, mar. 1995.

8 LIMA, M.H.P.; TELLES, F.J.S.; MACEDO, B.A.; BENEVIDES, S.D. Elaboração de queijo de coalho a partir de leite pasteurizado e inoculado com Streptococcus thermophyllus/ e Lactobacillus bulgaricus: aspectos bioquímico e sensorial. Boletim do Centro de Pesquisa e Produção de Alimentos (CEPPA), Curitiba, v.16, n.1, p. 37-44, jan/jun. 1998. 
9 LOTUFO, T. Solução na mesa: novas categorias de alimentos revolucionam o cardápio e a saúde do brasileiro. ISTO É, São Paulo, n. 1555, p. 136-137, jul. 1999.

10 MONTEIRO, C.L.B. Técnicas de análise sensorial. 2.ed. Curitiba: Universidade Federal do Paraná/CEPPA, 1984. 101 p.

11 MORAES, M.A.C. Métodos de avaliação sensorial dos alimentos. Campinas: UNICAMP/Faculdade de Engenharia de Alimentos, 1985. $85 \mathrm{p}$

12 NORUSIS, M. J. SPSS 6.0 guide to data analysis. Chicago: PrenticeHall, 1993. 675 p.

13 STONE, H.; SIDEL, J.L. Sensory evaluation practices. New York: Academic Press, 1993. 308 p.

14 TRONCO, V. M. Aproveitamento do leite e elaboração de seus derivados na propriedade rural. Guaíba: Agropecuária, 1996. 146 p.

15 WAKELING, I.N.; MACFIE, H.J. Designing consumer trials balanced for first and higher orders of carry-over effect when only a subset of k samples from t may be tested. Food Quality and Preference, v.6, p.299-308, 1995.

16 ZHANG, D.; MAHONEY, A.W. Iron fortification of process cheddar cheese. Journal of Dairy Science, v.74, n.2, p.353-358, 1991.

\section{AGRADECIMENTOS}

- Ao Albion Laboratories pela doação do aminoácido quelato (Ferrochel); - A UPEDA Setor de Laticínios e ao Laboratório de Controle de Qualidade de Alimentos, pertencentes ao Centro de Formação de Tecnólogos de Bananeiras (CFT) da UFPB - Campus IV, Bananeiras/PB, pelo apoio concedido na realização das análises. 